\title{
Non Spore-Forming Bacteria: Sterility and Ultrastructure Study
}

\author{
R.M. Hannah, C.A. Brantner, J.P. Burans, and R.K. Pope
}

Electron Microscopy Laboratory, National Biodefense Analysis and Countermeasures Center, Battelle National Biodefense Institute, 8300 Research Plaza, Fort Detrick, MD 21702.

Live organisms from containment laboratories must be inactivated with a valid protocol, or subsequently sterility tested prior to removal from containment and worked with in a lower level of containment. For bioforensic analysis, many specimens that are imaged using electron microscopy (EM) come from BSL2, BSL-3 and BSL-4 containment laboratories. Current literature documents many procedures for fixation of pathogenic organisms, but very few actually document sterility after fixation. For safety and bioforensic analysis, it has been necessary to identify the minimum times required to inactivate vegetative bacteria while preserving ultrastructure for EM. Recent information from this lab demonstrates that most bacterial endospores are inactivated in less than 240 minutes in Universal fixative (4\% paraformaldehyde/1\% glutaraldehyde (Brantner, et al. in press). Because spores are the most resistant form of bacteria, the data was useful in developing timelines for the treatment of vegetative cells. It has been considered necessary to treat samples containing pathogenic bacteria for periods up to 21 days for inactivation. In addition to potentially causing damage to the ultrastructure of samples, this prolonged fixation period increases the time required to process bioforensic samples. There are many protocols in the literature for inactivating bacterial samples, but they are either of long duration, or utilize harsh chemicals that destroy ultrastructure for subsequent analysis. This study demonstrates that all vegetative bacterial species, resuspended in fixative and tested, were successfully inactivated in 5 minutes. To standardize the laboratory protocols, the same samples were tested for 5 , $15,30,60,120$, and 240 minutes.

This study demonstrates the amount of time required to inactivate vegetative bacterial cells of a number of bacterial species with 4\% paraformaldehyde1\% glutaraldehyde (Universal fixative); including Francisella tularensis LVS; Klebsiella pneumoniae Isolate 3; Listeria monocytogenes EGD-e; and Salmonella enterica LT2. Purified bacterial vegetative cells were enumerated and subsequently inactivated with universal fixative in $0.1 \mathrm{M}$ sodium cacodylate buffer for 5, 15, 30, 60, 120, and 240 minutes (Fig. 1). All treated bacterial preparations were then washed in buffer and subsequently sterility tested by plating $10 \%$ of the sample volume. All samples documented to be sterile were removed from containment laboratories and processed for EM imaging. Standard methods of sample processing were employed for ultrastructural analysis by transmission electron microscopy (TEM) and scanning electron microscopy (SEM).

It was demonstrated that fixation with universal fixative inactivated all vegetative cells for all species in as little as 5 minutes. This data demonstrates that samples containing vegetative bacterial cells can be brought out of containment suites after treatment for 240 minutes (time required to inactivate potential spores present) in universal fixative solution. Internal and external ultrastructure integrity of the bacterial cells is similar at 5, 15, 30, 60, 120 and 240 minutes (Fig. 2). Therefore, fixation for 240 minutes at room temperature in universal fixative solution is sufficient for the fixation and inactivation of samples containing vegetative bacterial cells, while maintaining good preservation of both external and internal ultrastructure for EM analyses. 


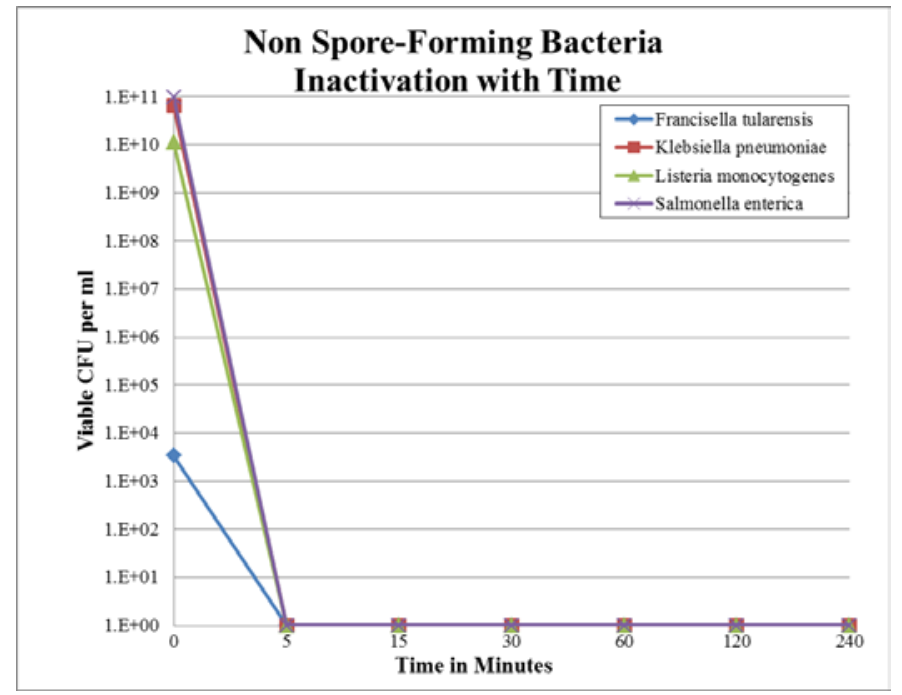

Figure 1. Graph of vegetative bacterial cell inactivation by resuspension in 4\% paraformaldehyde/1\% glutaraldehyde in $0.1 \mathrm{M}$ sodium cacodylate buffer. Triplicate tubes of Francisella tularensis, Klebsiella pneumoniae, Listeria monocytogenes, and Salmonella enterica were treated for times ranging from 5 minutes to 240 minutes.

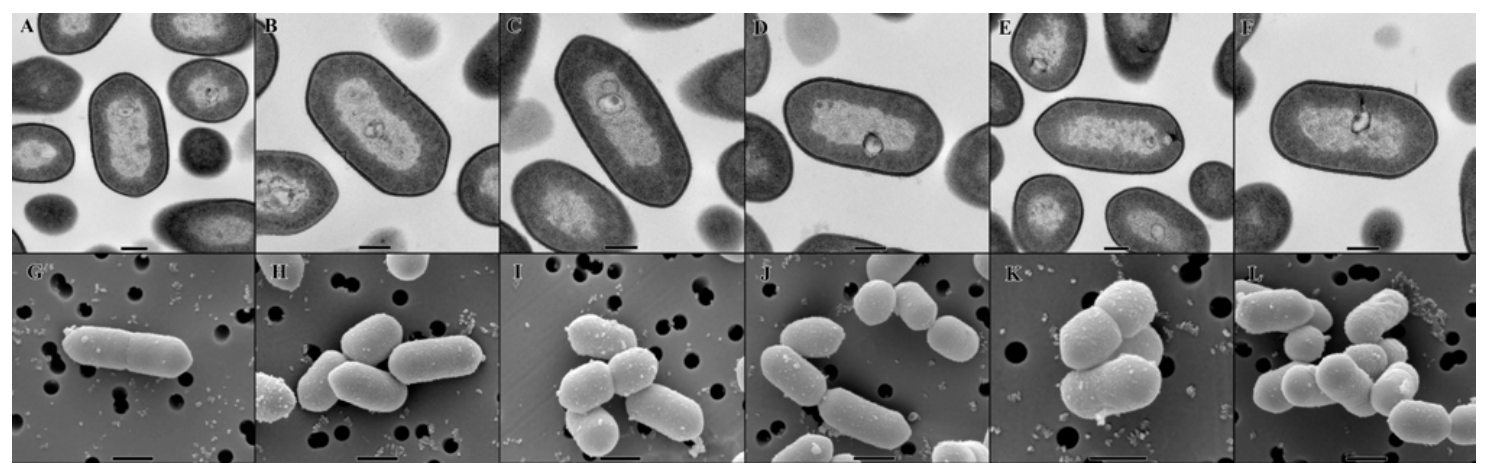

Figure 2. Transmission (A-F) and Scanning (G-L) electron micrographs of vegetative bacteria of Listeria monocytogenes fixed for different time periods. A, G) 5 minutes fixation, B, H) 15 minutes fixation, C, I) 30 minutes fixation, D, J) 60 minutes fixation, E, K) 120 minutes fixation, F,L) 240 minutes fixation. The internal and external ultrastructure of the vegetative bacterial cells remains very similar for each time point. A-F scale bar is $200 \mathrm{~nm}$. G-L scale bar is $500 \mathrm{~nm}$.

\section{References}

Brantner, CA, Hannah, RM, Pope, RK. 2013. Inactivation and Ultrastructure Analysis of Bacillus spp. and Clostridium perfringens Spores. Microscopy and Microanalysis (in press).

This work was funded under Agreement No. HSHQDC-07-C-00020 awarded by the Department of Homeland Security Science and Technology Directorate (DHS/S\&T) for the management and operation of the National Biodefense Analysis and Countermeasures Center (NBACC), a Federally Funded Research and Development Center. The views and conclusions contained in this document are those of the authors and should not be interpreted as necessarily representing the official policies, either expressed or implied, of the U. S. Department of Homeland Security. In no event shall the DHS, NBACC, or Battelle National Biodefense Institute (BNBI) have any responsibility or liability for any use, misuse, inability to use, or reliance upon the information contained herein. The Department of Homeland Security does not endorse any products or commercial services mentioned in this publication. 